\title{
Correction to: Differentiating focal nodular hyperplasia from hepatocellular adenoma: Is hepatobiliary phase MRI (HBP-MRI) using linear gadolinium chelates always useful?
}

Marion Roux $\odot,{ }^{1}$ Frederic Pigneur, ${ }^{2}$ Laurence Baranes, ${ }^{2,3}$ Julien Calderaro, ${ }^{4,3}$ Mélanie Chiaradia, ${ }^{2,3}$ Thomas Decaens, ${ }^{5,3}$ Sandrine Kastahian, ${ }^{6}$ Anaïs Charles-Nelson, ${ }^{6}$ Lambros Tselikas, ${ }^{2}$ Charlotte Costentin, ${ }^{5,3}$ Alexis Laurent, ${ }^{7,3}$ Daniel Azoulay, ${ }^{7,3}$ Ariane Mallat, ${ }^{5,3}$ Alain Rahmouni, ${ }^{2,3}$ Alain Luciani ${ }^{2,3,8}$

${ }^{1}$ Service de Radiodiagnostic et Radiologie interventionnelle, CHUV, Rue du Bugnon 46, 1011 Lausanne, Switzerland

${ }^{2}$ Groupe Henri Mondor Albert Chenevier, Imagerie Medicale, AP-HP, 94010 Creteil, France

${ }^{3}$ Faculte de Medecine de Creteil, Universite Paris Est Creteil, 94010 Creteil, France

${ }^{4}$ Groupe Henri Mondor Albert Chenevier, Pathology, AP-HP, 94010 Creteil, France

${ }^{5}$ Groupe Henri Mondor Albert Chenevier, Hepato-Gastroenterology Department, AP-HP, 94010 Creteil, France

${ }^{6}$ Groupe Henri Mondor Albert Chenevier, Unite de Recherche Clinique (URC), AP-HP, 94010 Creteil, France

${ }^{7}$ Groupe Henri Mondor Albert Chenevier, Liver Surgery, AP-HP, 94010 Creteil, France

${ }^{8}$ INSERM Unite U 955, Equipe 18, 94010 Creteil, France

Correction to: Abdom Radiol (2017) https://doi.org/10.1007/s00261-017-1377-z

The original version of this article unfortunately contained a mistake. There was an error in the last sentence of the summary and the last sentence of the discussion. It should read as "On HBP, all FNH appeared hyper- or iso-intense compared to adjacent liver while close to $97 \%$ of HCA appeared hypointense".

The original article can be found online at https://doi.org/10.1007/ s00261-017-1377-z.

Correspondence to: Marion Roux; email: marionroux@gmail.com 